\title{
On the growth of pebble-accreting planetesimals
}

\author{
Rico G. Visser and Chris W. Ormel

\begin{abstract}
Astronomical Institute Anton Pannekoek, University of Amsterdam, Science Park 904, PO box 94249, Amsterdam, The Netherlands e-mail: [r.g.visser; c.w.ormel] @uva.nl
\end{abstract}

Received 14 September 2015 / Accepted 10 November 2015

\section{ABSTRACT}

\begin{abstract}
Context. Pebble accretion is a newly discovered mechanism to quickly grow the cores of planets. In pebble accretion, gravity and gas drag conspire to yield large collisional cross sections for small particles in protoplanetary disks. However, before pebble accretion commences, aerodynamic deflection may act to prevent planetesimals from becoming large, because particles tend to follow gas streamlines.

Aims. We derive the planetesimal radius where pebble accretion is initiated and determine the growth timescales of planetesimals by sweep-up of small particles.

Methods. The equation of motion for a pebble, including gas drag and gravitational interactions, was integrated in three dimensions at distances of 1,3 , and $10 \mathrm{AU}$ from the star. We obtained the collision efficiency factor as the ratio of the numerically obtained collisional cross section to the planetesimal surface area, from which we obtained the growth timescales. Integrations were conducted in the potential flow limit (steady, inviscid) and in the Stokes flow regime (steady, viscid).

Results. Only particles of stopping time $t_{\mathrm{s}} \ll t_{\mathrm{X}}$ where $t_{\mathrm{X}} \approx 10^{3} \mathrm{~s}$ experience aerodynamic deflection. Even in this case, the planetesimal's gravity always ensures positive collision factors. The planetesimal radius where growth proceeds slowest is $\approx 100 \mathrm{~km}$ (less for colder disks) corresponding to interactions shifting from the geometric to the Safronov focusing regime. For particles $t_{\mathrm{s}} \gg t_{\mathrm{X}}$ pebble accretion only commences after this phase and is characterized by a steep drop in growth timescales. At 1 AU, growth timescales are shorter than the disk lifetime for pebbles larger than $0.03 \mathrm{~cm}$. The planetesimal radius $R_{\mathrm{PA}}$ where pebble accretion commences increases with disk orbital radius. At distances beyond $\sim 10 \mathrm{AU}$, sweep-up growth times are always longer than $10 \mathrm{Myr}$, while in the inner disk $(\$ 3 \mathrm{AU})$ the viability of the sweep-up scenario is determined by the outcome of pebble-planetesimal collisions in the geometric regime. We present analytical fits for the collision efficiency factors and the minimum planetesimal radius $R_{\mathrm{PA}}$ required for pebble accretion.
\end{abstract}

Key words. planets and satellites: formation - methods: numerical - protoplanetary disks - minor planets, asteroids: general hydrodynamics

\section{Introduction}

Pebble accretion (PA) is an accretion mechanism involving both gravitational and dissipative (gas drag) forces. Thus, PA covers interactions between a large body (planetesimal or planet) and small particles in the presence of gas. During their accretion, pebbles settle in the gravitational field of the large body, just like the buildup of sediments in seas or rivers ${ }^{1}$. This is in contrast to gas-free encounters, which rely on hitting the surface of the large body. As first demonstrated by Ormel \& Klahr (2010), PA rates can be much higher than the corresponding gas-free limit, since cross sections can become as large as the Hill sphere (Lambrechts \& Johansen 2012). If there is a massive pebble reservoir, this virtually eradicates the timescale problem that (giant) planet formation faces in the classical scenario of planetesimal accretion. PA is especially attractive because protoplanetary disks are inferred to harbor large amounts of pebble-sized particles, as suggested by radio observations (Andrews et al. 2009; Ricci et al. 2010; Testi et al. 2014). Furthermore, a strong concentration of these particles will trigger streaming instabilities, which leads to gravitationally bound objects that can produce

\footnotetext{
1 For this reason, Ormel \& Klahr (2010) referred to these interactions as "settling" and the corresponding parameter space where they occur as the "settling regime". We use this phrasing as a synonym with "pebble accretion", coined by Lambrechts \& Johansen (2012).
}

planetesimals (Youdin \& Goodman 2005; Johansen et al. 2009) the seeds required for the PA mechanism.

Recent works have advanced the PA theory and applied the concept to planet formation in the solar system and exoplanetary systems (Bitsch et al. 2015). Compared to planetesimals, pebbles are quite mobile and drift toward the central star (Weidenschilling 1977a). Therefore, PA is not an isolated problem, but requires global solutions for the pebble density in the disk as a function of time (Lambrechts \& Johansen 2014; Birnstiel et al. 2012; Krijt et al. 2016). How PA proceeds also depends on the size distribution of large bodies (planetesimal, protoplanets); and it is essential for reproducing the solar system architecture that only the largest bodies in the distribution profit from PA (Kretke \& Levison 2014; Levison et al. 2015). PA ceases when planets become massive enough to alter the gas structure of the protoplanetary disk - the pebble isolation mass which, applied to the solar system, is consistent with the heavy element contents of the giant planets (Lambrechts et al. 2014). In the inner solar system, PA has been invoked to explain the small size of Mars (Morbidelli et al. 2015) and it has been applied to explain the asteroid size distribution (Johansen et al. 2015b).

Under certain conditions, PA results in high accretion rates. But that does not necessarily imply that PA is efficient, as particles may drift past the (proto)planet before they can be accreted (Ormel \& Kobayashi 2012; Chambers 2014; Guillot et al. 2014; Sato et al. 2015). In particular, Guillot et al. (2014) calculated 
the probability of a collision for a particle traversing a planetesimal belt. This resulted in complex dependencies on the model parameters, but this filtering by planetesimals was generally seen to be most efficient for large planetesimals (protoplanets, operating in the settling regime) or for small planetesimals (which profit from a high ratio of surface area to mass) and for weak turbulence levels (which confines particles to the midplane). On the other hand, intermediate-sized planetesimals collect solids only inefficiently. In addition, Guillot et al. (2014) highlighted the importance of hydrodynamic flow effects, where small particles are so tightly coupled that they follow the streamlines of the gas, avoiding accretion by planetesimals (Michael \& Norey 1969; Whipple 1972; Slinn 1976; Sekiya \& Takeda 2003; Sellentin et al. 2013). Physically, aerodynamic deflection occurs when the particle stopping time $t_{\mathrm{s}}$ (which equals the particle momentum divided by the gas drag force) becomes shorter than the time it takes to cross the large body; that is, when the Stokes number ${ }^{2}$

$\mathrm{St}=\frac{t_{\mathrm{s}} v_{\mathrm{hw}}}{R}$

becomes lower than unity, where $R$ is the planetesimal radius and $v_{\text {hw }}$ the headwind velocity of the gas that the planetesimal faces and with which the tightly coupled particles approach it.

Guillot et al. (2014) conjectured that this aerodynamic deflection suppresses accretion of small particles by planetesimals. However, planetesimals do exert a gravitational force on the pebbles, which may mitigate the effect. By numerically integrating the pebble equation of motion, it is the goal of this work to quantify how severe the suppression becomes. We further calculate the mass-doubling timescales for planetesimals embedded in a sea of pebbles, identify the planetesimal size where growth experiences its key bottleneck, and the size where PA commences (these two sizes do not necessarily coincide).

We carry out integrations for both Stokes (high viscosity or Reynolds number $\mathrm{Re} \ll 1$ ) flow and inviscid (potential) flow. A caveat is that only steady flow patterns are considered, whereas planetesimals in the protoplanetary disk typically have Reynolds number $\gg 1$, implying that the flow structure is turbulent. However, by carrying out integrations for both the $\mathrm{Re} \ll 1$ and the inviscid limits, we anticipate to bracket the uncertainty in the results to some extent.

We show that by virtue of gravitational settling, planetesimal accretion rates are always higher than zero, even for the smallest grains (although in that case the collisional cross section will be much smaller than the geometrical cross section). These results agree with the Stokes flow integrations by Johansen et al. (2015b). Physically motivated expressions are presented that fit our numerical results, which are valid as long as Keplerian shear effects are unimportant (i.e., small planetesimals or small particles). In particular, we derive a critical stopping time of $\approx 10^{3} \mathrm{~s}$, above which particles avoid aerodynamic deflection.

The plan of the paper is as follows. In Sect. 2 we discuss the model setup and assumptions, including the adopted disk model and the dynamics of gas and pebbles, followed by the numerical methods. In Sect. 3 we present the collisional efficiency factors and growth timescales as a function of planetesimal size, pebble size, and location in the disk. In Sect. 4 we present analytical fitting expressions to our numerical data. In Sect. 5 we discuss some implications of our results, and we list our key conclusions in Sect. 6.

\footnotetext{
2 We note that the Stokes number defined here differs from what is usually adopted in the astrophysical literature, where it is instead identified with the $\tau_{\mathrm{s}}=t_{\mathrm{s}} \Omega$ where $\Omega$ is the local orbital frequency.
}

\section{Model setup and assumptions}

\subsection{Disk model}

The temperature and density profiles for the protoplanetary disk follow from the minimum mass solar nebula (MMSN; Weidenschilling 1977b; Hayashi et al. 1985),

$T(r)=300 \mathrm{~K}\left(\frac{r}{1 \mathrm{AU}}\right)^{-1 / 2}$,
$\Sigma(r)=1700 \mathrm{~g} \mathrm{~cm}^{-2}\left(\frac{r}{1 \mathrm{AU}}\right)^{-3 / 2}$,

with $r$ the distance from the star. If we assume hydrostatic equilibrium and an isothermal temperature profile in the vertical direction, the density profile is a Gaussian:

$\rho_{\mathrm{g}}(r, z)=\frac{\Sigma(r)}{H \sqrt{2 \pi}} \exp \left[-\frac{1}{2}\left(\frac{z}{H}\right)^{2}\right]$,

with $z$ the vertical distance and the scale height $H=c_{\mathrm{s}} / \Omega_{0}, \Omega_{0}$ the local Keplerian frequency and $c_{\mathrm{s}}=\sqrt{k_{\mathrm{b}} T / \bar{m}}$, the isothermal sound speed, $k_{\mathrm{b}}$ Boltzmann's constant, and $\bar{m}=2.34 \times 10^{-24} \mathrm{~g}$ the mean molecular weight. Since the gas in the disk is partially pressure supported, it rotates at speeds slightly below the Kepler speed $\left(v_{k}\right)$. The planetesimal thus faces a headwind of magnitude (Nakagawa et al. 1986)

$v_{\mathrm{hw}}=\frac{c_{\mathrm{s}}^{2}}{2 v_{k}} \frac{\partial \log P}{\partial \log r}=5780 \mathrm{~cm} \mathrm{~s}^{-1}$,

with $P(r)$ the pressure of the gas. For power-law profiles of $T$ and $\Sigma$ the headwind velocity is independent of disk orbital radius $r$. In Eq. (5) that the MMSN profile has been adopted for convenience; more realistic disk models give rise to different profiles than Eqs. (2) and (3). For example, the hydrodynamical simulations of Bitsch et al. (2015), which include radiative transport, result in a headwind velocity that is lower than Eq. (5). In Sect. 3.3 we briefly consider alternative disk profiles. We note, however, that the dimensionless fit formulas that we present in Sect. 4 cover every disk profile.

\subsection{The pebble equation of motion}

To integrate pebble streamlines we chose a local frame comoving with the planetesimal. The planetesimal was assumed to be massive and to move on a circular Keplerian orbit. The equation of motion in three dimensions is given by (Ormel \& Klahr 2010)

$$
\frac{\mathrm{d} \boldsymbol{v}}{\mathrm{d} t}=\left(\begin{array}{c}
2 \Omega_{0} v_{y}+3 \Omega_{0}^{2} x \\
-2 \Omega_{0} v_{x} \\
0
\end{array}\right)-\frac{G M}{r^{3}}\left(\begin{array}{l}
x \\
y \\
z
\end{array}\right)+\boldsymbol{F}_{\mathrm{drag}}
$$

where $v$ is the velocity, $G$ Newton's gravitational constant, $M$ the mass of the planetesimal, $(x, y, z)$ the coordinates of the pebble in the local frame, and $\boldsymbol{F}_{\text {drag }}$ the drag force. The first terms of Eq. (6) consist of the Coriolis acceleration and tidal accelerations, respectively, while the second term describes the planetesimal gravity. We note that the stellar acceleration term in the $z$-direction $\left(-\Omega_{0}^{2} z\right)$ has been omitted because this would otherwise render vertical impact parameters infinite for a laminar gas flow adopted here.

We used two steady prescriptions for the gas flow in the vicinity of the planetesimals (see Fig. 1): inviscid potential flow 


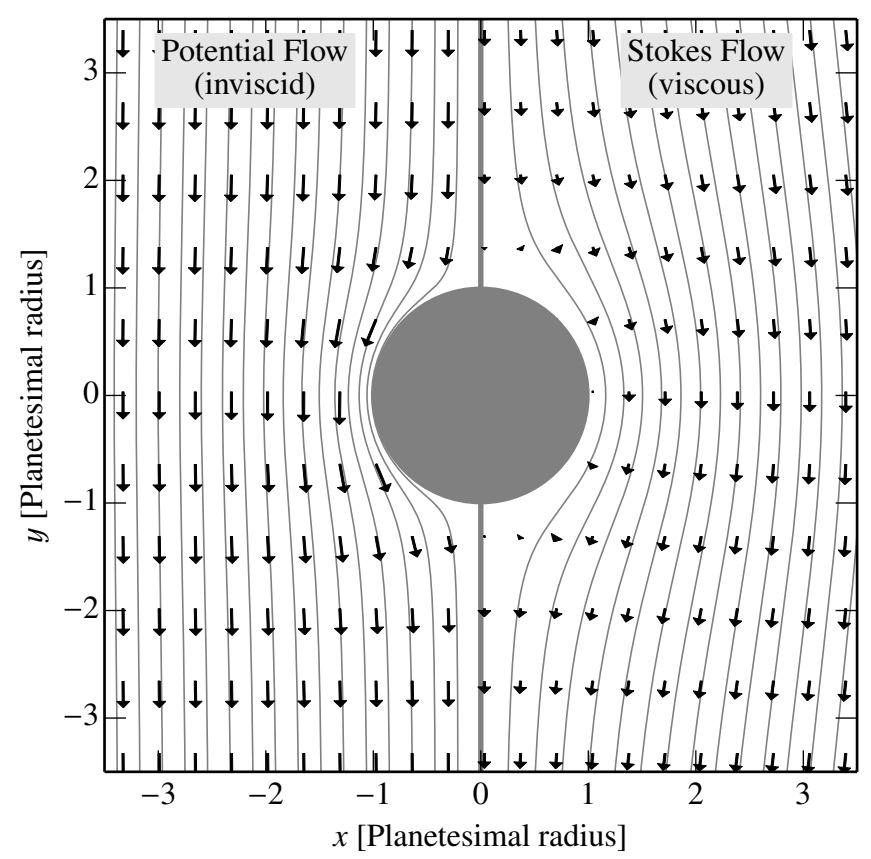

Fig. 1. Velocity vectors and gas streamlines around a sphere (planetesimal) for potential flow (left half; Eq. (7)) and Stokes flow (right half; Eq. (8)) as seen in the frame of the sphere.

and (highly viscid) Stokes flow. The velocity of potential flow past a sphere reads, in spherical coordinates (Batchelor 1967)

$\boldsymbol{v}_{\mathrm{Pot}}=v_{\mathrm{hw}} \cos \theta\left(1-\frac{R^{3}}{r^{3}}\right) \boldsymbol{e}_{r}+v_{\mathrm{hw}} \sin \theta\left(1+\frac{R^{3}}{2 r^{3}}\right) \boldsymbol{e}_{\theta}$,

with $\theta$ the angle between the direction of the unperturbed flow (here: $-\boldsymbol{e}_{y}$ ) and a point $\mathbf{x}$ in the local frame and $R$ is the planetesimal radius. Similarly, the Stokes flow solution reads (Batchelor 1967)

$\boldsymbol{v}_{\mathrm{Stk}}=v_{\mathrm{hw}} \cos \theta\left(1+\frac{R^{3}}{2 r^{3}}-\frac{3 R}{2 r}\right) \boldsymbol{e}_{r}+v_{\mathrm{hw}} \sin \theta\left(1-\frac{R^{3}}{4 r^{3}}-\frac{3 R}{4 r}\right) \boldsymbol{e}_{\theta}$,

see Fig. 1. Compared to Stokes flow, the potential solution does not contain $\propto 1 / r$ terms. Stokes flow therefore modifies the unperturbed flow even at large distances from the planetesimal, which is a consequence of viscosity. In addition, in Stokes flow the gas velocity is identically 0 at $r=R$, whereas in potential flow $v_{r}=0$, but $v_{\theta} \neq 0$ (see Fig. 1 ).

To these solutions, we added the shear-corrected gas velocity, rendering the combined flow velocity of the gas to become

$\boldsymbol{v}_{\mathrm{g}}(x)=\boldsymbol{v}_{\text {flow }}-\frac{3}{2} \Omega_{0} x \boldsymbol{e}_{y}$,

with $\boldsymbol{v}_{\text {flow }}$ either the Stokes or the potential flow solution. Strictly, the addition in Eq. (9) is not self-consistent: it would, for example, violate the irrotational assumption used in deriving Eq. (7). However, as the scales on which Eqs. (7) and (8) and the Keplerian shear apply are very different $\left(R \Omega_{0} \ll v_{\text {hw }}\right)$, we expect the error to be negligible and irrelevant to the results of this work.

The gas drag force $\boldsymbol{F}_{\text {drag }}$ felt by the particles reads

$\boldsymbol{F}_{\text {drag }}=-\frac{\boldsymbol{v}_{\mathrm{p}}-\boldsymbol{v}_{\mathrm{g}}}{t_{\mathrm{s}}}$

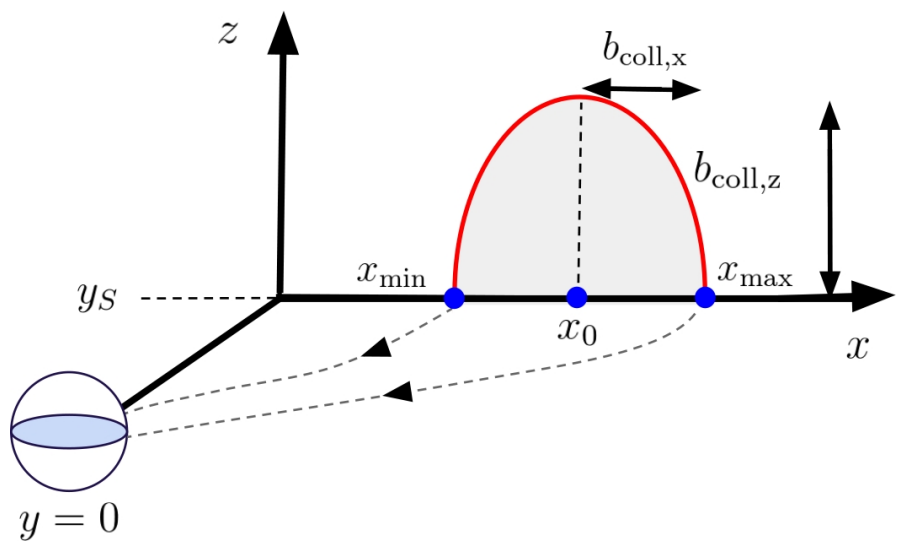

Fig. 2. Sketch of the procedure we used to obtain the collisional cross section. In the comoving frame, the planetesimal resides at the origin $(y=0)$ and pebbles start at a distance $y_{\mathrm{s}}$ far enough to be unperturbed by the gravity of the planetesimal. Integrations are first carried out in the $x$-direction, and the impact parameter $b_{\text {coll, } x}$ is determined from the first and last trajectory that hits the planetesimal $\left(x_{\min }\right.$ and $\left.x_{\max }\right)$. Starting at the average of these two coordinates $x_{0}$, the integrations are extended to the $z$-direction to obtain the vertical impact parameter $b_{\text {coll, }, z}$.

where $\boldsymbol{v}_{\mathrm{p}}$ is the velocity of the particle, $\boldsymbol{v}_{\mathrm{g}}$ the gas velocity, and $t_{\mathrm{s}}$ the stopping time for a spherical particle with radius $s$ (Whipple 1972; Weidenschilling 1977a):

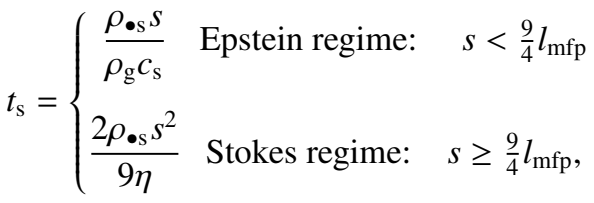

with $l_{\text {mfp }}$ the mean free path of the gas molecules, $\rho_{\bullet, s}$ the internal particle density taken to be $1 \mathrm{~g} \mathrm{~cm}^{-3}$ and $\eta$ the dynamic viscosity. As our largest particle sizes are $30 \mathrm{~cm}$, these are the relevant stopping time regimes.

\subsection{Numerical integrations}

We numerically integrated the particle streamlines with the Runge-Kutta-Fehlberg variable step method (RKF45). This integration method uses variable steps and compares a fourth-order solution with a fifth-order solution to determine the next step size (Fehlberg 1969; Eshagh 2005; Ormel \& Klahr 2010). We used a relative error tolerance of $10^{-8}$, small enough to ensure convergence. The goal of the integrations is to determine the collisional cross-sections of pebbles of a radius $s$ hitting a planetesimal of radius $R$. This is achieved by integrating the pebble equation of motion, Eq. (6), starting at a position $\left(x_{\mathrm{S}}, y_{\mathrm{S}}\right)$ from the planetesimal, see Fig. 2. At this point, the initial velocities read:

$v_{x}=v_{x, \infty}=-\frac{2 v_{\mathrm{hw}} \tau_{\mathrm{s}}}{1+\tau_{\mathrm{s}}^{2}}$,

$v_{y}\left(x_{\mathrm{S}}\right)=v_{y, \infty}-\frac{3}{2} \Omega_{0} x_{\mathrm{S}}=-\frac{v_{\mathrm{hw}}}{1+\tau_{\mathrm{s}}^{2}}-\frac{3}{2} \Omega_{0} x_{\mathrm{S}}$,

with $v_{x, \infty}, v_{y, \infty}$ the (unperturbed) radial and azimuthal drift velocities of the pebble (Weidenschilling 1977a), $-\frac{3}{2} \Omega_{0} x_{\mathrm{S}}$ the correction for Keplerian shear, and $\tau_{\mathrm{s}}=\Omega_{0} t_{\mathrm{s}}$ the dimensionless stopping time. The initial starting line $y=y_{\mathrm{S}}$ was chosen to be far enough from the planetesimal to ensure that the gravitational force at the starting location is much smaller than the gas drag 
A\&A 586, A66 (2016)

Table 1. Overview of the parameter space and the corresponding numerical results at given orbital distance $r$.

\begin{tabular}{|c|c|c|c|c|c|c|c|c|}
\hline$r$ & Flow & $\begin{array}{l}S \\
{[\mathrm{~cm}]}\end{array}$ & $\tau_{\mathrm{s}}$ & $f_{\text {coll,min }}$ & $\begin{array}{l}t_{\mathrm{gr}, \max } \\
{[\mathrm{yr}]}\end{array}$ & $\begin{array}{l}R_{\mathrm{gr}, \text { max }} \\
{[\mathrm{km}]}\end{array}$ & $\begin{array}{l}R_{\mathrm{PA}} \\
{[\mathrm{km}]}\end{array}$ & Comments \\
\hline \multirow[t]{14}{*}{$1 \mathrm{AU}$} & pot & 0.01 & $8.5 \times 10^{-6}$ & 0.001 & $1.8 \times 10^{9}$ & 40 & 50 & \\
\hline & stk & 0.01 & $8.5 \times 10^{-6}$ & 0.005 & $6.9 \times 10^{7}$ & 10 & 20 & \\
\hline & pot & 0.03 & $2.5 \times 10^{-5}$ & 0.03 & $1.4 \times 10^{8}$ & 80 & 90 & \\
\hline & stk & 0.03 & $2.5 \times 10^{-5}$ & 0.03 & $2.4 \times 10^{7}$ & 30 & 40 & \\
\hline & pot & 0.1 & $8.5 \times 10^{-5}$ & 0.3 & $1.7 \times 10^{7}$ & 130 & 150 & \\
\hline & stk & 0.1 & $8.5 \times 10^{-5}$ & 0.2 & $7.7 \times 10^{6}$ & 50 & 50 & \\
\hline & pot & 0.3 & $2.5 \times 10^{-4}$ & 0.8 & $5.3 \times 10^{6}$ & 110 & 130 & \\
\hline & stk & 0.3 & $2.5 \times 10^{-4}$ & 0.5 & $3.3 \times 10^{6}$ & 50 & 60 & \\
\hline & pot & 1 & $8.5 \times 10^{-4}$ & 1.0 & $2.9 \times 10^{6}$ & 90 & 110 & \\
\hline & stk & 1 & $8.5 \times 10^{-4}$ & 0.8 & $2.7 \times 10^{6}$ & 40 & 50 & \\
\hline & pot & 3 & $2.5 \times 10^{-3}$ & 1.0 & $2.4 \times 10^{6}$ & 80 & 90 & \\
\hline & stk & 3 & $2.5 \times 10^{-3}$ & 1.0 & $2.4 \times 10^{6}$ & 70 & 80 & \\
\hline & pot & 10 & $2.5 \times 10^{-2}$ & 1.0 & $2.2 \times 10^{6}$ & 80 & 150 & \\
\hline & pot & 30 & $2.3 \times 10^{-1}$ & 1.0 & $2.2 \times 10^{6}$ & 80 & 300 & \\
\hline \multirow[t]{10}{*}{$3 \mathrm{AU}$} & pot & 0.01 & $4.4 \times 10^{-5}$ & 0.75 & $1.2 \times 10^{8}$ & 110 & 120 & \\
\hline & pot & 0.03 & $1.3 \times 10^{-4}$ & 1.0 & $6.5 \times 10^{7}$ & 90 & 110 & \\
\hline & pot & 0.1 & $4.4 \times 10^{-4}$ & 1.0 & $5.0 \times 10^{7}$ & 80 & 130 & \\
\hline & pot & 0.3 & $1.3 \times 10^{-3}$ & 1.0 & $4.6 \times 10^{7}$ & 80 & 150 & \\
\hline & pot & 1 & $4.4 \times 10^{-3}$ & 1.0 & $4.5 \times 10^{7}$ & 80 & 200 & \\
\hline & pot & 3 & $1.3 \times 10^{-2}$ & 1.0 & $4.4 \times 10^{7}$ & 80 & 250 & \\
\hline & pot & 10 & $4.4 \times 10^{-2}$ & 1.0 & $4.4 \times 10^{7}$ & 80 & 350 & \\
\hline & pot & 30 & $5.7 \times 10^{-2}$ & 1.0 & $4.4 \times 10^{7}$ & 50 & 200 & $3 \rho_{\mathrm{g}}$, see Fig. 5 \\
\hline & pot & 30 & $1.3 \times 10^{-1}$ & 1.0 & $4.4 \times 10^{7}$ & 80 & 450 & \\
\hline & pot & 30 & $1.3 \times 10^{-1}$ & 1.0 & $4.4 \times 10^{7}$ & 30 & 150 & $T=90 \mathrm{~K}$, see Fig. 5 \\
\hline \multirow[t]{7}{*}{$10 \mathrm{AU}$} & pot & 0.01 & $2.7 \times 10^{-4}$ & 1.0 & $1.3 \times 10^{7}$ & 80 & 150 & \\
\hline & pot & 0.03 & $8.1 \times 10^{-4}$ & 1.0 & $1.3 \times 10^{7}$ & 70 & 200 & \\
\hline & pot & 0.1 & $2.7 \times 10^{-3}$ & 1.0 & $1.2 \times 10^{7}$ & 80 & 250 & \\
\hline & pot & 0.3 & $8.1 \times 10^{-3}$ & 1.0 & $1.2 \times 10^{7}$ & 80 & 350 & \\
\hline & pot & 1 & $2.7 \times 10^{-2}$ & 1.0 & $1.2 \times 10^{7}$ & 80 & 500 & \\
\hline & pot & 3 & $8.1 \times 10^{-2}$ & 1.0 & $1.2 \times 10^{7}$ & 80 & 600 & \\
\hline & pot & 10 & $2.7 \times 10^{-1}$ & 1.0 & $1.2 \times 10^{7}$ & 80 & 750 & \\
\hline
\end{tabular}

Notes. Columns denote the orbital disk radius $r$, the adopted flow solution (potential or Stokes), the particle radius $s$, the dimensionless stopping time $\tau_{\mathrm{s}}=t_{\mathrm{s}} \Omega$, the minimal collision factor $f_{\text {coll,min }}$ obtained from the results shown in Fig. 3, the maximum growth timescale $t_{\mathrm{gr}, \text { max }}$ obtained from the results shown in Fig. 4 (unless denoted otherwise in the comment section), the corresponding planetesimal radius $R_{\mathrm{gr}, \max }$, and the planetesimal radius where pebble accretion starts $R_{\mathrm{PA}}$.

force. Specifically, we adopted

$y_{\mathrm{S}}=\max \left(50 R, 10^{3} \sqrt{\frac{G M}{v_{\mathrm{hw}} / t_{\mathrm{s}}}}\right)$.

Starting in the midplane $(z=0)$, we first varied $x_{\mathrm{S}}$ and determined whether the integration results in a hit or a miss. Thus, we searched for the positions $\left\{x_{\min }, x_{\max }\right\}$ separating hits from misses. The condition for a hit is given by

$\sqrt{x^{2}+y^{2}+z^{2}}-R \leq 0$

We then determined the impact parameter as $b_{\text {coll, } x}=\left(x_{\max }-\right.$ $\left.x_{\min }\right) / 2$. By focusing our search around $x=x_{\min }, x_{\max }$ we ensured that the error in $b_{\text {coll }, x}$ is at most $1 \%$. Next, we integrated in the $z$-direction starting at the midpoint $\left(x_{0}=\left(x_{\max }+\right.\right.$ $\left.x_{\min }\right) / 2, y_{\mathrm{S}}, z=0$ ) to determine the impact parameter in the vertical direction $b_{\text {coll,z }}$ (see Fig. 2). We assumed that the impact geometry is ellipsoidal and that the impact area (at $y=y_{\mathrm{S}}$ ) is $A=\pi b_{\mathrm{coll}, x} b_{\mathrm{coll}, z}$. The corresponding pebble flux is $F=A \hat{\boldsymbol{n}} \cdot \boldsymbol{v}=$ $\pi b_{\text {coll }, x} b_{\text {coll }, z} v_{y}\left(x_{0}\right)$, with $\hat{\mathbf{n}}$ the unit vector perpendicular to $A$. Multiplied by $\rho_{\mathrm{p}}$, the spatial density of pebbles in the midplane, this gives a total accretion rate $\dot{M}$. As in the geometric limit the flux is $\pi R^{2} v_{\mathrm{hw}}$, the collisional efficiency is therefore

$f_{\text {coll }}=\frac{\pi b_{\mathrm{coll}, x} b_{\mathrm{coll}, z} v_{y}\left(x_{0}\right)}{\pi R^{2} v_{\mathrm{hw}}}$.

In terms of $f_{\text {coll }}$, the accretion rate is

$\dot{M}=\pi R^{2} v_{\mathrm{hw}} \rho_{\mathrm{p}} f_{\text {coll }}$.

\section{Results}

Results for $f_{\text {coll }}$ are obtained as function of particle radius $s$, planetesimal radius $R$, disk orbital radius (1, 3, and $10 \mathrm{AU})$ and flow pattern (Stokes or potential). We adopted internal densities of $\rho_{\bullet}=1 \mathrm{~g} \mathrm{~cm}^{-3}$ for both planetesimals and pebbles. Results of the integrations are given in Table 1 and in Figs. 3 and 4.

\subsection{Collision efficiencies}

For small planetesimals, the collisional cross section equals the geometric cross section, $f_{\text {coll }} \approx 1$. However, the Stokes number (Eq. (1)) decreases for increasing planetesimal sizes. When it reaches unity, aerodynamic deflection can become important as the particle will adjust to the flow on a timescale $\left(t_{\mathrm{s}}\right)$ that is 
R. G. Visser and C. W. Ormel: On the growth of pebble-accreting planetesimals
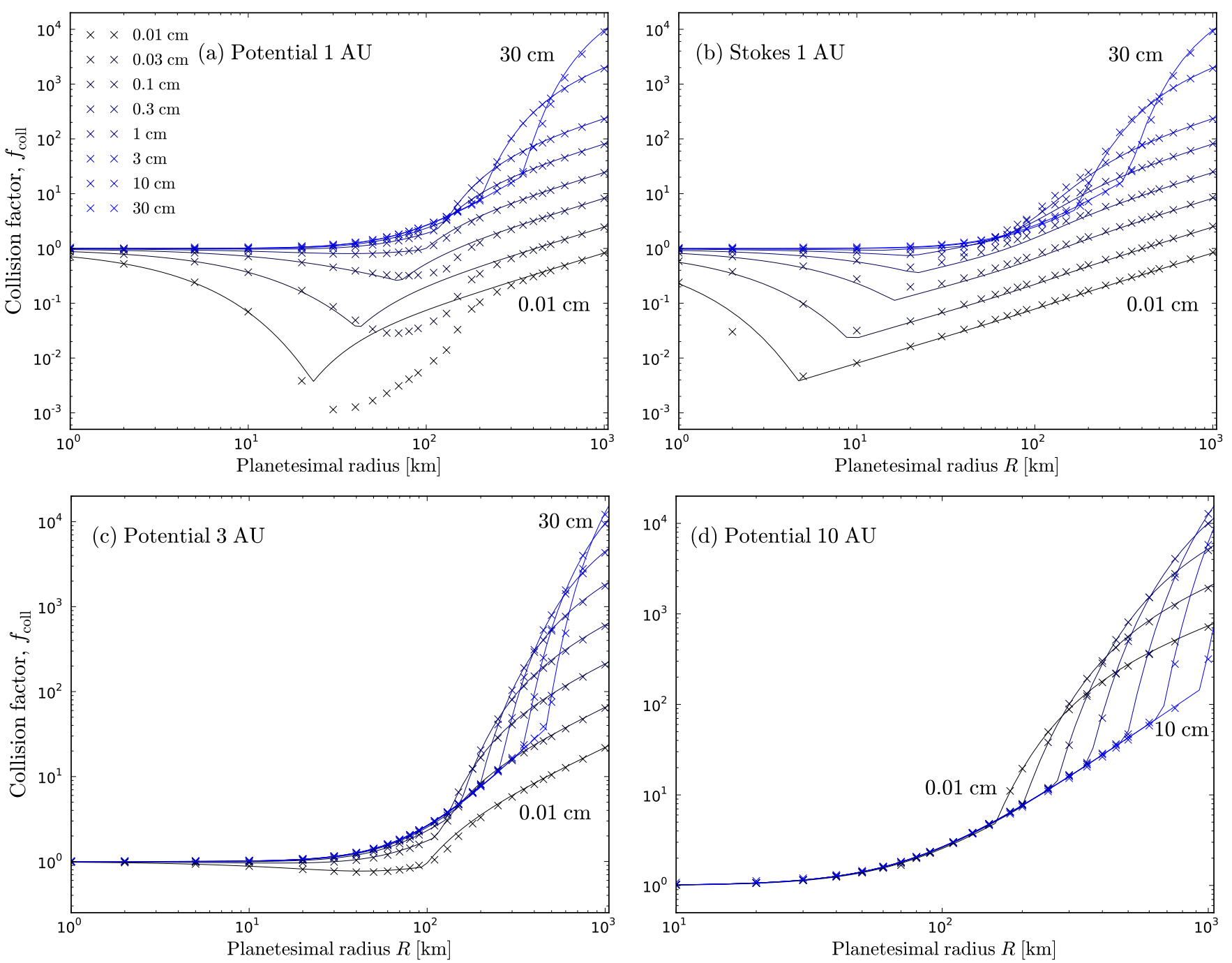

Fig. 3. Collision factor $f_{\text {coll }}$ as a function of planetesimal radius $R$ for a constant internal density $\rho_{\mathbf{\bullet}}=1 \mathrm{~g} \mathrm{~cm}^{-3}$. Crosses indicate numerical data, while solid lines are fits. The color scale ranges from dark to light blue for increasing particle radius. a) Results obtained with the (inviscid) potential solution (Eq. (7)) at $1 \mathrm{AU}$ orbital distance from the star. For a small planetesimal size, the collision factor is approximately geometrical $\left(f_{\text {coll }} \approx 1\right)$. However, for small pebble sizes, aerodynamic deflection results in a significant drop of $f_{\text {coll }}$ (see Table 1 for the minimum collision efficiency, $\left.f_{\text {coll,min }}\right)$. b) Results with the (highly viscid) Stokes flow solution (Eq. (8)). Settling occurs at smaller sizes due to the increase in encounter time. c) Results at $3 \mathrm{AU}$ orbital distance show a shallow barrier only for the lowest particle size. d) At $10 \mathrm{AU}$ a barrier is no longer present because of the reduced gas density.

shorter than the crossing time $R / \Delta v$. Furthermore, for small particles, (below $0.1 \mathrm{~cm}$ ) the two-body gravitational force is weak. Consequently, the collision factor becomes (much) smaller than unity. This is consistent with the non-gravitational hydrodynamical simulations by Sekiya \& Takeda (2003); under these conditions, particles hardly hit the planetesimal. For our smallest size of $10^{-2} \mathrm{~cm}$, collisional deflection factors reach a minimum of $10^{-3}$ for the potential case and slightly higher for the Stokes case. In addition, the minimum in Stokes flow occurs at smaller planetesimals. On the other hand, Figs. $3 \mathrm{a}$ and $\mathrm{b}$ show that $f_{\text {coll }}$ never reaches zero. The minimum value for the collision factor, $f_{\text {coll,min }}$, is listed in Table 1 .

This difference between the two flow patterns can be explained as follows. In both cases the radial velocity is zero at the surface of the planetesimal. However, whereas the angular component is zero in the viscous case (zero slip boundary), it is of the order of the headwind velocity for potential flow. For the potential case this explains the deep barrier that is seen in Fig. 3a: particles feel a centrifugal force (of the order of $\sim v_{\mathrm{hw}}^{2} / R$ ) that competes with the gravitational attraction. On the other hand, for Stokes flow the almost vanishing azimuthal velocities means that the crossing times become significantly longer than $R / v_{\mathrm{hw}}$, which has two consequences: (i) aerodynamic deflection becomes effective at higher Stokes number (smaller planetesimal size for fixed $t_{\mathrm{s}}$ ); and (ii) gravity has more time to act, promoting accretion by settling. As a result, the minimum of the collisional efficiencies for Stokes flow occurs at a smaller planetesimal radius.

In Table 1 two modes can be identified. (i) For pebbles smaller than $0.1 \mathrm{~cm}$ hydro effects operate to slow down particles within the time pebbles encounter the planetesimal. The collision factor steadily decreases, with $f_{\text {coll,min }}$ possibly $\ll 1$ due to aerodynamic deflection, before settling interactions reverse the decline. (ii) Larger pebbles $(s>0.1 \mathrm{~cm}$ at $0.1 \mathrm{AU})$ never experience aerodynamic deflection. Instead, we see a gradual increase of $f_{\text {coll }}$, characteristic of the smooth transition to Safronov gravitational focusing. Collision factors then increase with planetesimal size. Eventually, gravity becomes strong enough to trap 

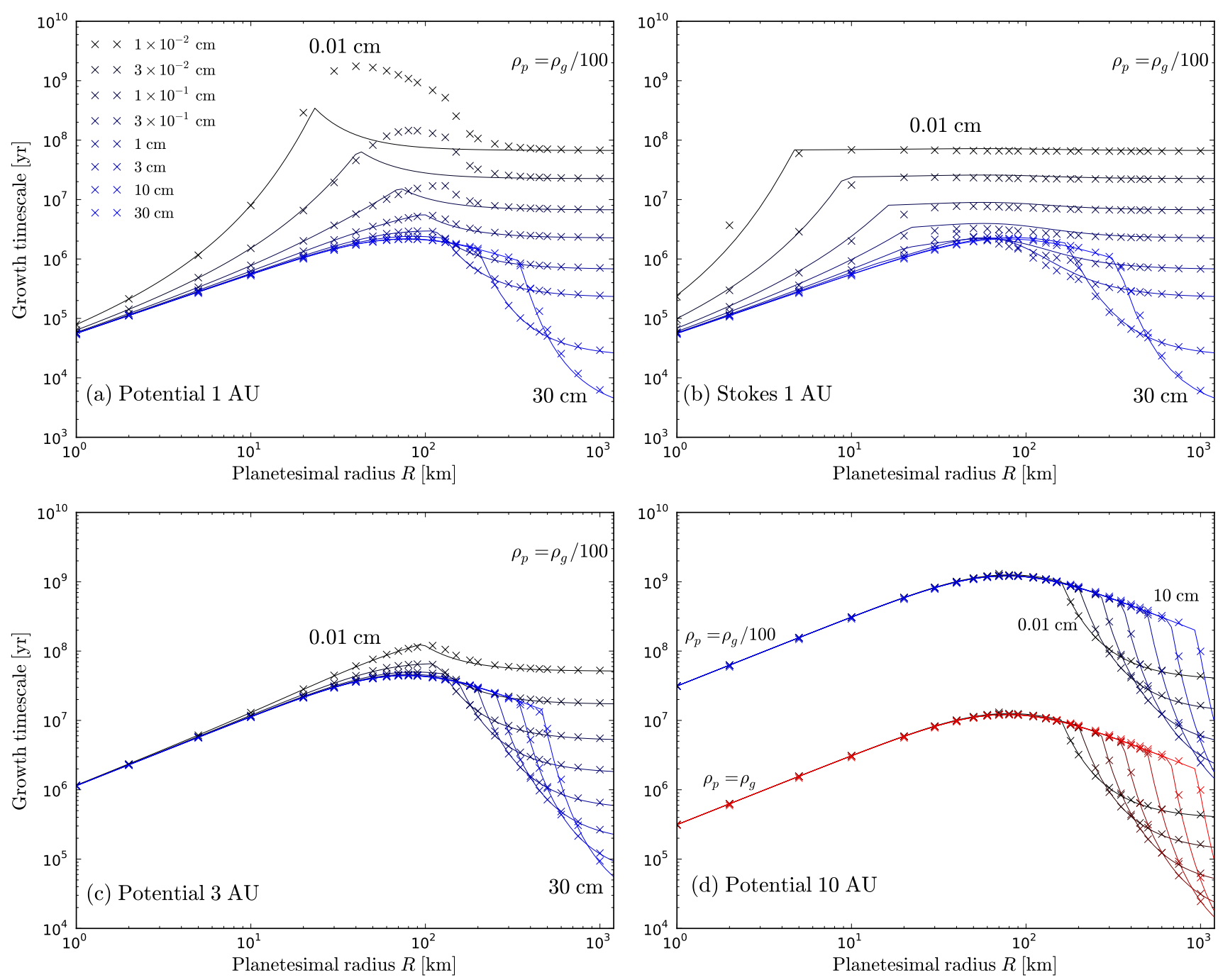

Fig. 4. Growth timescale $t_{\text {growth }}$ as function of planetesimal radius $R$ for a constant internal density $\rho_{\bullet}=1 \mathrm{~g} \mathrm{~cm}^{-3}$. Crosses indicate numerical data, while the solid lines are fits. The color scale extends from dark blue to light blue, transitioning from lowest particle radius to highest particle radius. a) $t_{\text {growth }}$ obtained with the potential flow solution at $1 \mathrm{AU}$ orbital distance and a pebble density of $\rho_{\mathrm{p}}=\rho_{\mathrm{g}} / 100$, showing maxima at $R_{\mathrm{gr}, \max } \sim 100 \mathrm{~km}$. b) $t_{\text {growth }}$ with the Stokes flow solution at $1 \mathrm{AU}$ orbital distance and a pebble density of $\rho_{\mathrm{p}}=\rho_{\mathrm{g}} / 100$, showing early settling and flattening out of the curves. c) $t_{\text {growth }}$ with the potential flow solution at $3 \mathrm{AU}$ orbital distance and a pebble density of $\rho_{\mathrm{p}}=\rho_{\mathrm{g}} / 100$. d) $t_{\mathrm{growth}}$ with the potential flow solution at $10 \mathrm{AU}$ orbital distance and a pebble density of $\rho_{\mathrm{p}}=\rho_{\mathrm{g}} / 100$ (top, blue) and a pebble density of $\rho_{\mathrm{p}}=\rho_{\mathrm{g}}$ (bottom, red).

pebbles in the Hill sphere, initiating the onset of pebble accretion, which expresses itself as a steep upturn in $f_{\text {coll }}$.

As was explained above, the onset of pebble accretion also depends on disk radius. The integrations at $3 \mathrm{AU}$ and $10 \mathrm{AU}$ with the potential flow solution are shown in Figs. $3 \mathrm{c}$ and d. In Fig. $3 \mathrm{c}$ only the smallest particle shows a barrier (see Table 1). For larger particle radii, the collision efficiency factors start in the geometric regime, smoothly make the transition to the Safronov regime, and then enter the settling regime. Since stopping times increase with disk radius and with particle radius, the onset of pebble accretion occurs at larger planetesimal radius, explaining the prolonged duration of ballistic encounters.

\subsection{Growth timescale for sweep-up}

Equation (17) defines the timescale for the planetesimal to $e$-fold its mass:

$t_{\text {growth }}=\frac{M}{\dot{M}}=\frac{4 \rho_{\bullet} R}{3 v_{\text {hw }} \rho_{\mathrm{p}} f_{\text {coll }}}$.
The growth timescales for the same parameter space as our results for $f_{\text {coll }}$ are shown in Fig. 4 and listed in Table 1. The density of pebbles is taken $\rho_{\mathrm{p}}=\rho_{\mathrm{g}} / 100$, the typical solid-to-gas ratio. In Fig. 4d, however, we also consider the case of equal pebble and gas density. Figures $4 \mathrm{a}$ and $\mathrm{b}$ show growth timescales using the potential and Stokes flow solutions at 1 AU orbital distance. The time needed for the planetesimal to increase its mass by a factor $e$ increases for small planetesimal size $R$ up to a maximum $R_{\text {gr,max }}$. In the potential case, this maximum is centered around $R_{\mathrm{gr}, \max } \sim 100 \mathrm{~km}$ (see Table 1 ). For the simulations with the Stokes flow pattern, the maximum shifts to smaller pebble sizes.

The features presented in Fig. 4 reflect those of Fig. 3. In the geometrical regime, growth timescales increase since the dependency of planetesimal mass on radius is cubic and the dependency on the impact parameter is quadratic. For pebble sizes $\lesssim 0.1 \mathrm{~cm}$, the increase in growth time is especially dramatic due to the aerodynamic deflection discussed in Sect. 3.1. Pebbles $\gtrsim 10 \mathrm{~cm}$ never experience aerodynamic deflection, but instead enter the Safronov regime (as was discussed 


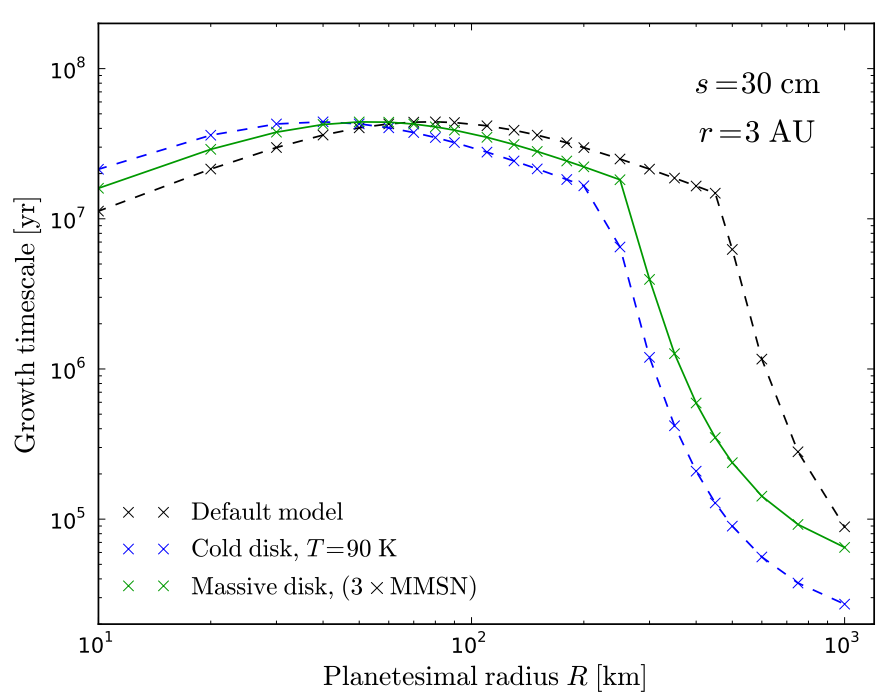

Fig. 5. Growth timescale $t_{\text {growth }}$ for a pebble of radius $s=30 \mathrm{~cm}$ as function of planetesimal radius $R$ at $3 \mathrm{AU}$. The black dashed curve shows the default model (as in Fig. 4). The other curves consecutively give the effects of a colder disk resulting in a headwind of $v_{\mathrm{hw}}=28 \mathrm{~cm} \mathrm{~s}^{-1}$ (blue dashed line) and a threefold increase in gas density (green curve) with a -0.5 power-law index for the surface density (Eq. (3)). The crosses represent numerical results and the solid (and dashed) lines represent fits.

in Sect. 3.1). Although Safronov focusing causes a decrease in growth timescale with planetesimal radius (runaway growth), growth timescales remain long. Nevertheless, growth inevitably transitions to the pebble accretion regime, which commences with a steep drop in $t_{\text {growth }}$.

At 3 and 10 AU orbital distances aerodynamic deflection is absent, except for pebbles smaller than $0.01 \mathrm{~cm}$ at $3 \mathrm{AU}$. For larger pebbles, the growth time initially decreases slowly (Safronov), but is followed by a sharp transition to pebble accretion (Figs. 4c and d). Because gas densities decrease with disk radius (Eq. (4)), $t_{\text {growth }}$ increases for most pebble radii. We see that in many cases peak growth timescales are much longer than the expected lifetimes of 6-10 Myr of gas-rich protoplanetary disks around low-to-intermediate-mass stars (Yasui et al. 2014; Pfalzner et al. 2014; Ribas et al. 2015). Planet formation by pebble sweep-up starting from small planetesimals would therefore be too slow. However, particle settling to the mid-plane of the disk (Youdin \& Lithwick 2007; Andrews et al. 2009) will increase pebble densities. In Fig. 4d we included the case that $\rho_{\mathrm{g}}=\rho_{\mathrm{p}}$, which reduces the timescales a hundred fold. Nevertheless, at $10 \mathrm{AU}$, the resulting peak growth timescales of $10^{7} \mathrm{yr}$ are still rather long.

\subsection{Varying the disk model}

Until now we have adopted the disk profile as given by Eqs. (2) and (3). In reality, however, there is no clear physical reason why a protoplanetary disk should be characterized by these MMSNlike profiles. We therefore considered the effect of changing the temperature and density. In Fig. 5 we show the growth timescale of a $30 \mathrm{~cm}$ particle at $3 \mathrm{AU}$ for a disk with a temperature of $90 \mathrm{~K}$ (green solid curve), which is half that of the standard model (black dashed curve). Such colder disk conditions may follow from more efficient cooling (Bitsch et al. 2015). The key consequence of the temperature drop is that it reduces the headwind of the disk (Eq. (5)) by a factor 2 . The reduced headwind increases the interaction timescale, providing more time for gravity to act. Thus, settling interactions now occur at a small planetesimal size $(200 \mathrm{~km}$ instead of $450 \mathrm{~km})$ and the peak growth times also shift to smaller planetesimal sizes $(\simeq 50 \mathrm{~km})$ because Safronov focusing also profits from a lower $v_{\mathrm{hw}}$. On the other hand, geometric encounters, which scale $\propto v_{\mathrm{hw}}$, are less effective, explaining the increase of the growth timescale at small $R$. Consequently, the peak growth timescale hardly changes; the growth time bottleneck remains but shift to smaller sizes.

The green line in Fig. 5 corresponds to the case of a more massive disk than in the default model. Here, we adopted a -0.5 exponent in the surface density expression of Eq. (3), which results in an increase of the gas density by a factor 3 . The pebble density $\rho_{\mathrm{p}}$ was kept the same as in the default model, however. Because of the shallower density profile, the headwind velocity drops slightly (to $40 \mathrm{~m} \mathrm{~s}^{-1}$ ), but the main effect of the enhanced gas density is that the $30 \mathrm{~cm}$ size particles have become aerodynamically smaller (lower $t_{\mathrm{s}}$ ). Increasing the pebble density by a factor of three, such that $\rho_{\mathrm{g}} / \rho_{\mathrm{p}}$ is back at 100 (not shown), causes the growth timescale to drop by a factor of three.

\section{Analysis: the onset of pebble accretion}

\subsection{Fitting expressions for ballistic and settling interactions}

Pebble accretion occurs when particles settle to the planetesimal at terminal velocities $v_{\text {settl }}=f_{\mathrm{g}} t_{\mathrm{s}}$, with $f_{\mathrm{g}}=G M / b^{2}$ the gravitational acceleration and $b$ the impact parameter. The equation that determines the impact parameter $b_{\text {set }}$ of these settling encounters is the cubic

$b^{2}\left(v_{\mathrm{hw}}+\frac{3}{2} \Omega b\right)=4 G M t_{\mathrm{s}}$

(Ormel \& Klahr 2010). This equation follows from equating the settling timescale $b / v_{\text {settl }}$ with the encounter timescale, $b / \Delta v$ where $\Delta v$, the approach velocity, is the term in braces in Eq. (19). The numerical factor 4 follows from the strongly coupled limit $\left(t_{\mathrm{s}} \rightarrow 0\right)$, which allows closed-form solutions (Ormel \& Klahr 2010). Since we consider only the onset of pebble accretion, the shear term, $\frac{3}{2} \Omega b$, can be ignored; then, $b_{\mathrm{set}}=\sqrt{4 G M t_{\mathrm{s}} / v_{\mathrm{hw}}}$.

At long stopping times interactions are too fast for settling because the interaction timescale $\sim b / \Delta v$ becomes shorter than the stopping time and particles never reach terminal velocities during the encounter. Consequently, Eq. (19) becomes invalid and pebble accretion inefficient for stopping times beyond a critical stopping time:

$t_{*}=\frac{4 G M}{v_{\mathrm{hw}}^{3}}$,

implying that pebble accretion cross sections are largest for particles of stopping time $t_{\mathrm{s}} \sim t_{*}$ (Lambrechts \& Johansen 2012).

For stopping times longer than $t_{*}$ particles experience the well-known (Safronov) gravitational focusing $b_{\mathrm{Saf}}=R \sqrt{1+\Theta}$, where $\Theta=\left(v_{\text {esc }} / v_{\text {hw }}\right)^{2}$ is the Safronov number (Safronov 1972) with $v_{\text {esc }}=\sqrt{2 G M / R}$ the surface escape velocity of the body. We refer to these encounters as "ballistic" 3 because the encounter

3 Since the parameter space is enormous, gas drag-mediated accretion can be (sub-)categorized into several regimes. Originally, Ormel \& Klahr (2010) identified "settling", "hyperbolic" (=ballistic), and threebody (more massive particles that experience little gas drag) encounters. Lambrechts \& Johansen (2012) divided the settling regime into Hill accretion (for massive bodies) and a Bondi regime (used here). Guillot et al. (2014) subdivided the ballistic regime into "geometric" (where $f_{\text {coll }} \approx 1$ ), "Safronov" $\left(f_{\text {coll }}>1\right)$, and "hydro", where $f_{\text {coll }} \ll 1$ due to aerodynamic deflection. 


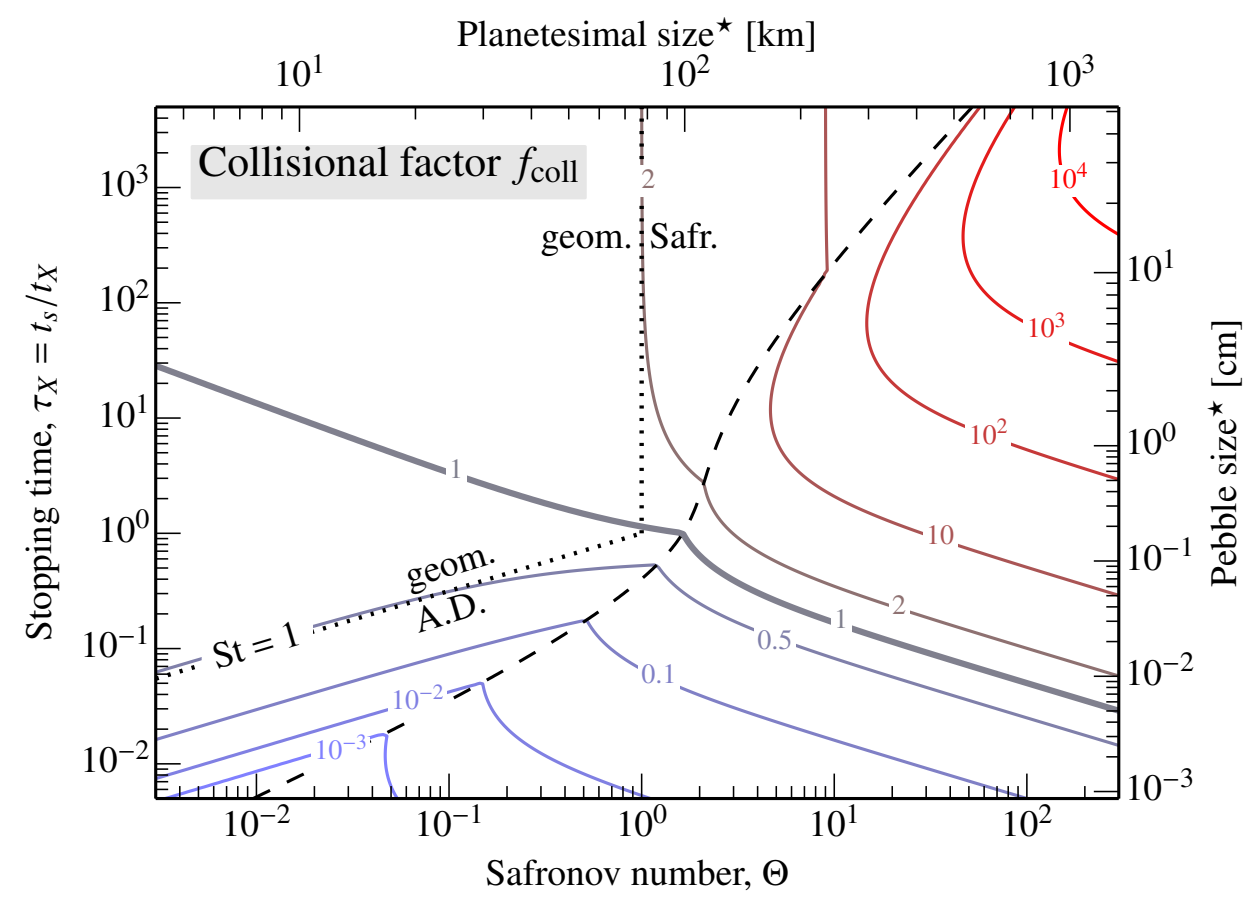

Fig. 6. Contour plot of $f_{\text {coll }}$ (Eq. (21)) for potential flow as a function of Safronov number ( $x$-axis) and dimensionless stopping time $\tau_{\mathrm{X}}=t_{\mathrm{s}} / t_{\mathrm{X}}(y$-axis). The former is a proxy for the planetesimal size, whereas $\tau_{\mathrm{X}}$ is a measure of the particle aerodynamical size. The dashed curve gives the transition between ballistic and settling interactions. The dotted lines $\Theta=1$ and $\mathrm{St}=$ 1 separate the geometric regime (where $f_{\text {coll }} \approx 1$ ) from the Safronov (where $\Theta>1$ ) and aerodynamic deflection (A.D., where St $<1)$ regimes. At small Stokes number, aerodynamic deflection suppresses accretion because these particles couple too well to the gas for ballistic interactions to be effective. Particles of $\tau_{\mathrm{X}} \lesssim 1$ experience aerodynamic deflection before entering the settling regime, while $\tau_{\mathrm{X}} \gtrsim 1$ particles experience Safronov focusing before entering the pebble accretion regime. ( $\star$ : the conversion of $\tau_{X}, \Theta$ to physical parameters has been conducted for the default parameters at 1 AU.)

Table 2. Parameters for the collision factor for the settling and the ballistic regimes, see Eq. (21).

\begin{tabular}{lcccc}
\hline \hline Flow & $f_{\text {coll, }, 0}$ & $\mathrm{St}_{*}$ & $a$ & $b$ \\
\hline \multicolumn{5}{c}{ Settling interactions } \\
Stokes & $2 \Theta \mathrm{St}$ & $2 \Theta+4+4 / \Theta$ & 2.26 & 0.61 \\
Potential & $2 \Theta \mathrm{St}$ & $2 \Theta$ & 2.26 & 0.61 \\
\hline \multicolumn{5}{c}{ Ballistic interactions } \\
Stokes & $1+\Theta$ & 1 & 3.24 & -0.86 \\
Potential & $1+\Theta$ & 1 & 0.78 & -0.89 \\
\hline
\end{tabular}

Notes. Here, $f_{\text {coll, }, 0}$ and $\mathrm{St}_{*}$ are given by physical considerations and $a$ and $b$ are fit parameters.

that grazes the object determines the impact parameter. This is in contrast to the settling mode, where $b_{\text {set }}$ is independent of the physical size $R$ - the defining characteristic of pebble accretion. When encounters become too long, gas drag acts to diminish the efficacy of ballistic interactions: projectiles follow gas streamlines. Specifically, gas drag prevents small particles from colliding with the target when the stopping time is much shorter than $R / v_{\mathrm{hw}}$, or St $\ll 1$. Such particles never ballistically collide with a planetesimal.

In conclusion, hydrodynamical effects suppress ballistic encounters below $\mathrm{St} \sim 1$, whereas for larger Stokes numbers (stopping times) settling encounters vanish. We can describe both behaviors with a fitting function of the form

$f_{\text {coll }}=f_{\text {coll }, 0} \exp \left[a\left(\frac{\mathrm{St}}{\mathrm{St}_{*}}\right)^{b}\right]$,

where $f_{\text {coll, }, 0}$ is the uncorrected collision efficiency, and $a$ and $b$ are fit parameters that depend on the adopted flow pattern. The critical Stokes number $\mathrm{St}_{*}$ is fixed at 1 for ballistic encounters and $2 \Theta$ (corresponding to $t_{*}$ in Eq. (20)) for settling interactions in potential flow. For Stokes flow we adopted a different prescription for $\mathrm{St}_{*}$. The parameters are listed in Table 2.

These fits were kept relatively simple and do not cover all situations. For example, in the zero-gravity limit $(\Theta=0) f_{\text {coll }}$ does not become identically 0 below a critical Stokes number (Slinn 1976). Generally, $f_{\text {coll }}$ in the ballistic regime depends on the Reynolds number of the flow. For turbulent flows, furthermore, the suppression of $f_{\text {flow }}$ at low $\mathrm{St}$ is much diminished (Homann et al. 2015, see Sect. 5.2). In the settling regime, $f_{\text {coll }}$ is consistent with earlier results (Ormel \& Klahr 2010; Ormel \& Kobayashi 2012) barring three-body effects ${ }^{4}$.

Contours of $f_{\text {coll }}$ for potential flow are plotted in Fig. 6 as function of Safronov number $\Theta$ and

$\tau_{\mathrm{X}} \equiv \Theta^{1 / 2} \mathrm{St}=\frac{t_{\mathrm{s}}}{t_{\mathrm{X}}}=\frac{t_{\mathrm{s}}}{1337 \mathrm{~s}}\left(\frac{\rho_{\bullet}}{1 \mathrm{~g} \mathrm{~cm}^{-3}}\right)^{1 / 2}$,

where $t_{\mathrm{X}}=\sqrt{3 / 8 \pi G \rho_{\bullet}}$ depends only on the planetesimal internal density. When $\rho_{\bullet}$ is constant, $\tau_{X}$ can therefore be identified with the pebble aerodynamical properties (in contrast to St, which is a mix of planetesimal and pebble properties), allowing us to convert the dimensionless axes of Fig. 6 to physical parameters. At high Stokes number (large $\tau_{X}$ ) and small $\Theta$, ballistic encounters operate in the geometric regime (top left). With decreasing $\tau_{\mathrm{X}}$ (smaller particle sizes) interactions suffer aerodynamic deflection as the Stokes number decreases. Collision efficiency factors then rapidly decrease, until the point where $f_{\text {coll }}$ becomes determined by settling encounters (dashed curve). Nevertheless, for $\tau_{X} \lesssim 1$ settling accretion rates are always modest even for $\Theta \gg 1$ (bottom right corner). On the other hand, for $\tau_{\mathrm{X}} \gtrsim 1$ particles, the journey to pebble accretion involves the Safronov regime. This already ensures that $f_{\text {coll }}$ increases above unity, but $f_{\text {coll }}$ is especially boosted by pebble accretion after the dashed curve has been crossed (top right).

The fits of Table 2 provide a good match to the data, see Fig. 3. We note that $\mathrm{St}_{*}$ in the Stokes flow fit in the settling regime becomes $\gg 1$ for small planetesimals $(\Theta \ll 1)$. This extends the power-law solution of the settling regime and results in "flat" growth timescales (see Fig. 4b). The physical reason behind this change is that for low-impact parameters $(b \ll R)$ encounter times are much longer than the $b_{\text {set }, 0} / v_{\text {hw }}$ that is assumed in the derivation of $t_{*}$ (Eq. (20)) because the transverse

4 Three-body effects become important for stopping times longer than the orbital period $\left(t_{\mathrm{s}} \gtrsim \Omega^{-1}\right)$ and for large planetesimals (protoplanets) for which $t_{*} \gtrsim \Omega^{-1}$. In these cases Eq. (21) and Table 2 are not applicable. 
velocity $\left(v_{\theta}\right)$ approaches zero (see Fig. 1). In fact, in Stokes flow the interaction time increases with decreasing cross section: $t_{\mathrm{enc}} \sim R / \delta v \sim R^{2} / b v_{\mathrm{hw}}$, where $\delta v$ is the transverse velocity at a distance $\delta r$ from the sphere and $\delta r \sim b$ (cf. Johansen et al. $2015 b$ ). Continuing this order-of-magnitude analysis, the collision factor is still given by $b_{\text {set }, 0}^{2}$, but the critical Stokes number (where $t_{\mathrm{s}}=t_{\mathrm{enc}}$ ) is now $\mathrm{St}_{*} \sim \Theta^{-1}$. This explains the expression for $\mathrm{St}_{*}$ adopted in Table 2.

In the potential case a similar analysis results in $\mathrm{St}_{*} \simeq 1+2 \Theta$, but we found that the offset made little difference. More importantly, the fit expressions for potential flow fail at very small impact parameters (see Fig. 3a). The reason is that centrifugal forces due to the flow curvature overwhelm gravitational forces, that is, particles are propelled away from the planetesimals, unless they collide head-on. This results in another reduction of the collision efficiency compared to the fit in Fig. 3a.

\subsection{Critical stopping time for accretion}

From Fig. 6 it is clear that the value of $\tau_{X}$ is a key factor in the growth of planetesimals. In Fig. 6, particles of the same stopping time move from left to right in Fig. 6 as the planetesimal size increases. Starting from this point $\left(\Theta \ll 1\right.$ and $\left.f_{\text {coll }}=1\right)$ and increasing the planetesimal size $(\Theta)$, two growth modes can be identified:

1. $\tau_{\mathrm{X}} \ll 1$ particles become affected by aerodynamic deflection (St $\lesssim 1)$ before gravitational focusing starts $(\Theta>1)$. With increasing planetesimal size (decreasing Stokes number), collision factors become significantly suppressed, $f_{\text {coll }} \ll 1$, stalling the growth of the planetesimal. At some point, settling interactions will take over from ballistic encounters, after which $f_{\text {coll }}$ increases with $\Theta$ in the settling regime. The settling regime accretion rates for $\tau_{\mathrm{X}} \ll 1$ particles are nevertheless modest: $f_{\text {coll }} \propto M$ ( neutral growth).

2. $\tau_{\mathrm{X}} \gg 1$ particles experience gravitational focusing $(\Theta>$ 1) first and are never affected by aerodynamic deflection. Initially, $f_{\text {coll }}$ increases due to (classical) Safronov gravitational focusing, $f_{\text {coll }} \simeq \Theta$, but when $t_{\mathrm{s}} \sim t_{*}$ at a Safronov number of $\Theta \sim \tau_{\mathrm{X}}^{2 / 3}$, collision efficiencies exponentially increase as interactions transition to the settling regime. Collisional focusing factors jump from $\sim \tau_{\mathrm{X}}^{2 / 3}$ to $\sim \tau_{\mathrm{X}}^{4 / 3}$ over a relatively short range in $\Theta^{5}$. For longer stopping times, the jump in $f_{\text {coll }}$ associated with entering the pebble accretion regime is therefore more dramatic - but occurs at a higher planetesimal mass.

\section{Discussion}

\subsection{Viability of the incremental growth of small planetesimals}

In our calculations of the growth timescale we assumed perfect sticking of pebbles with planetesimals. However, dust collisional experiments often give a plethora of collisional outcomes, ranging from sticking, bouncing, erosion and catastrophic destruction (Blum \& Wurm 2008; Güttler et al. 2010). This raises the question whether the reported growth timescales are realistic. Windmark et al. (2012) concluded that the (positive) mass transfer at small projectile sizes transitions to (negative) erosion if

\footnotetext{
5 The scalings follow by evaluating $f_{\text {coll }}$ in the ballistic and settling regime for $\mathrm{St}=\mathrm{St}_{*}$, which occurs at $\Theta=\left(\tau_{\mathrm{X}} / 2\right)^{2 / 3}$. In reality, particles enter the settling regime at even lower $\Theta$ as a result of the exponential tail, and the jump is even larger (see Eq. (24)).
}

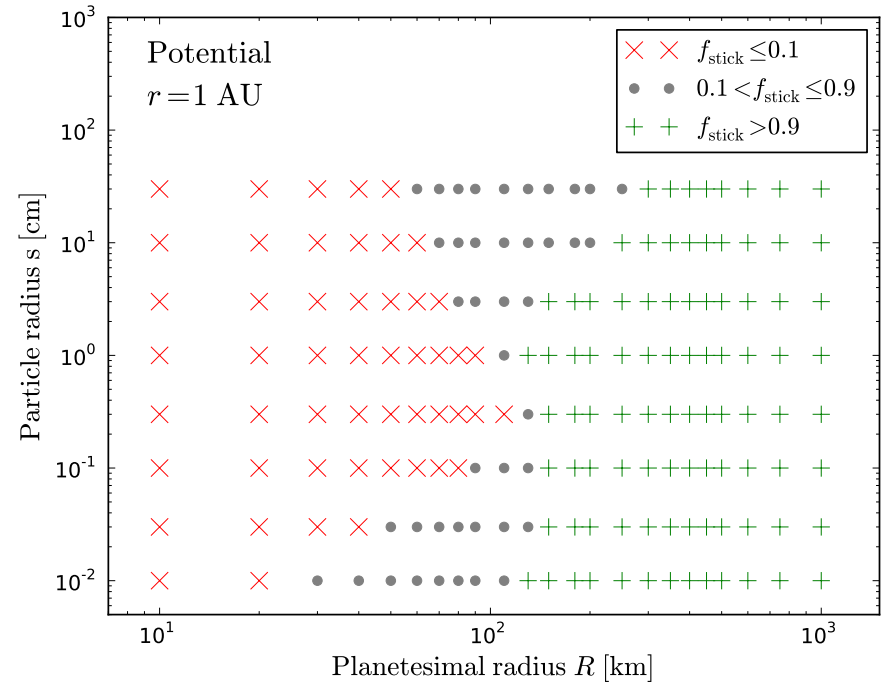

Fig. 7. Scatter plot of planetesimal radius $R$ vs. particle radius $s$ for potential flow at $1 \mathrm{AU}$ from the star. The scatter plot shows the result of streamline integrations of the same parameter space as in Fig. 3a, assuming particles bounce off the surface. After 10 bounces, particles are considered accreted. The red crosses represent a sticking fraction of $f_{\text {stick }}<0.1$, the gray dots of $0.1<f_{\text {stick }} \leq 0.9$ and the green crosses $f_{\text {stick }}>0.9$.

the projectile size becomes larger than $\sim 0.1 \mathrm{~mm}$, and Krijt et al. (2015) argued that erosion of large, fluffy planetesimals replenishes disks with a fresh reservoir in small grains.

To assess the dependence of the collisional physics on the growth rates, we considered a simulation where particles bounce off the surface of the planetesimal. We adopted the same parameters as in Fig. 3a (1 AU, potential flow) and, following Johansen et al. (2015b), adopted a coefficient of restitution of $50 \%$ for a single bounce. Particles were considered accreted after they had bounced ten times with the planetesimal. Clearly, the accretion cross section including bouncing will not exceed the previously determined collisional cross section. Let this ratio be called $f_{\text {stick }}$. Hence, $f_{\text {stick }}=0$ implies that all particles bounce off (to become re-entrained in the gas flow), whereas $f_{\text {stick }}=1$ means that all colliding particles return to the planetesimal (because of its gravity). The results of these bouncing simulations are presented in Fig. 7, where symbols indicate the value of $f_{\text {stick}}$. The parameter space where the sticking fraction is low (red crosses: $f_{\text {stick }} \leq 0.1$ ) approximately correspond to the geometrical regime. On the other hand, in the Safronov regime $(\Theta>1)$, corresponding to a planetesimal size of $100 \mathrm{~km}$, accretion becomes more effective, while in the settling regime $f_{\text {stick }}=1$ is guaranteed. At small particle radii $(s=0.01 \mathrm{~cm}$ and $s=0.03 \mathrm{~cm})$, the sticking fraction again increases because the few particles that accrete in this aerodynamic deflection regime do so by virtue of settling. All these findings are (qualitatively) consistent with Johansen et al. (2015b), who considered a Stokes flow.

These results therefore show that planetesimals below $100 \mathrm{~km}$ cannot grow merely by gravitational effects. However, the laboratory collision experiments that most resemble our case - small projectiles that are being fired onto a solid wall present a more positive view. Teiser \& Wurm (2009) studied collisions between centimeter dust projectiles and a steel plate with velocities ranging from 30 to $47 \mathrm{~m} \mathrm{~s}^{-1}$. This experiment shows that the accretion efficiency is positive (30\%). Furthermore, Meisner et al. (2013) studied the continuous impact of submillimeter $(0.1-1 \mathrm{~mm})$ dust particles onto larger objects. The results 
are consistent with the findings of Teiser \& Wurm (2009) and show that planetesimals indeed gain net mass with an accretion efficiency of $30 \%$ on average. Therefore, collision physics is not necessarily an impediment for the sweep-up growth scenario in the ballistic regime.

But are these loosely bound pebbles not simply blown off the surface of the planetesimals as the latter moves through the nebular gas? On Earth, lift forces arise because of variations in flow velocities and pressure near the ground. On Earth, as well on Mars, lifting of small grains is the first step toward saltation a process that shapes desert landscapes and causes dust storms. Modeling these effects, Shao \& Lu (2000) presented an empirical but physically motivated expression for the velocity required to initiate saltation (also known as the fluid threshold; Bagnold 1936):

$v^{*} \simeq 0.1 \sqrt{\frac{2 \rho_{\bullet, \mathrm{s}} f_{\mathrm{g}} s}{\rho_{\mathrm{g}}}+\frac{\gamma}{2 \rho_{\mathrm{g}} s}}$,

where $v^{*}$ is the threshold velocity for lifting (which must be compared to the headwind velocity $\left.v_{\mathrm{hw}}\right), f_{\mathrm{g}}$ is the gravitational acceleration and $\gamma \sim 0.1 \mathrm{~g} \mathrm{~s}^{-2}$ an empirical constant that represents the strength of the interparticle forces. The same forces operate on a rubble pile planetesimal moving through the nebular gas at velocities $v_{\mathrm{hw}}$. Therefore, when the magnitude of the lift force exceeds that of the gravity and interparticle forces, pebbles may be lifted from the planetesimal. Equation (23) suggests that small particles as well as large pebbles will remain bound because of sticking and high inertia, respectively. Nevertheless, for the relatively high densities in the inner disk $v^{*}$ may become lower than the planetesimal headwind velocity for small planetesimals ${ }^{6}$.

\subsection{Importance of the flow near the planetesimal}

In this work we have demonstrated that sweep-up growth timescales increase for particles below a critical stopping time of $t_{\mathrm{X}} \approx 10^{3} \mathrm{~s}$. In this case, St $\ll 1$ before Safronov focusing becomes important (Fig. 6). Under these conditions, we showed that there is some difference in collision efficiency factors between the potential and Stokes flow solutions. Which of the two adopted solutions is more realistic?

Without access to hydrodynamical simulations, we can only be descriptive. However, as the planetesimal Reynolds number is $\gg 1$, it may be argued that the potential flow solution - as unphysical as it is - is perhaps more applicable than the Stokes flow solution, which unrealistically alters the flow pattern at distances beyond several planetesimal radii (see our Fig. 1 and Beard \& Grover 1974). On the other hand, at very small impact parameters $\left(f_{\text {coll }} \ll 1\right)$ velocities close to the planetesimal surface must vanish, which renders the Stokes solution more appropriate. Continuing this reasoning, we may define a distance $\delta r$ from the surface of the planetesimal where the molecular diffusion timescale $(\delta r)^{2} / v$ equals the inertial transport time $v_{\mathrm{hw}} / R$. We then obtain $\delta r \sim R / \sqrt{\operatorname{Re}}$ (essentially the width of the boundary layer) to be the distance from the planetesimal beyond which the gas flow is better described by potential flow.

\footnotetext{
6 Care must be taken to extrapolate Eq. (23), which has been tailored for terrestrial conditions, towards the much rarefied nebular gas. Equation (23) must at least be modified in two points. First, it assumes a quadratic dependence on velocity of the gas drag law (as on Earth). Second, we can expect that small particles in the Epstein regime experience much reduced lift forces.
}

Another situation arises when the medium itself is turbulent. The gas in the protoplanetary disk, for one, is believed to be mildly turbulent due to hydrodynamic or magnetohydrodynamical instabilities (Balbus \& Hawley 1991). Recently, Homann et al. (2015) numerically determined the collision efficiencies as a function of Stokes number and turbulent intensity, finding a much shallower exponential decline (i.e., a fit parameter $a$ (see Table 2) much closer to zero). This means that the ballistic regime in Fig. 6 becomes gains in importance: the dashed line separating the geometric and aerodynamic deflection regimes shifts down.

At $\sim 3$ AU particles of stopping time $t_{\mathrm{s}} \ll t_{\mathrm{X}}$ correspond to $\mu \mathrm{m}$-grains. Since these particles are accreted at $f_{\text {coll }} \ll 1$ efficiencies, meteorites can only accrete trace amounts of them. While this is sufficient to explain the few presolar grains that are found in meteorites (Clayton \& Nittler 2004), it is clear that accretion of micron-size grains will not significantly contribute to the total mass. Therefore, the bulk of the matrix material found in chondrites must have been brought in through larger, aerodynamically more weakly coupled particles - for example, as dust-rimmed chondrules or aggregates of dust-rimmed chondrules (Cuzzi 2004; Ormel et al. 2008; Johansen et al. 2015a).

Finally, while we proposed a framework applicable to calculate accretion rates on planetesimals, it would be highly desirable to further refine our results by hydrodynamical simulations, which includes inertial particles that feel the gravity of the planetesimal ${ }^{7}$.

\subsection{Onset of pebble accretion}

Especially in the outer disk peak growth timescales can easily become longer than the disk lifetime. This means that the fast pebble accretion regime is not accessible by incremental growth processes - a situation exacerbated when pebbles are drifting very quickly inward and may be lost from the disk before the disk itself disappears. Therefore, a more attractive scenario for the growth of planets is to start from a planetesimal seed massive enough for interactions to fall in the pebble accretion regime. Such large planetesimals may be produced by gravitational instabilities involving pebble-size particles (for instance streaming instabilities; Johansen et al. 2007, 2009, 2012), be scattered from the inner disk, or be the collisional product of a population of small planetesimals. However, this threshold for the onset of pebble accretion $R_{\mathrm{PA}}$ shifts to progressively larger sizes for increasing disk orbital radii and increasing particle stopping times (Table 2).

We can obtain $R_{\mathrm{PA}}$ by equating $f_{\text {coll }}$ in the Safronov and settling regime. However, this is a transcendental equation in $R$. To still obtain an (approximate) closed-form expression, we assume that the transition occurs near the point where $t_{*} \approx t_{\mathrm{s}}$, yielding $R \approx v_{\mathrm{hw}} t_{\mathrm{X}}\left(t_{\mathrm{S}} / 2 t_{\mathrm{X}}\right)^{1 / 3}$. Modifying this expression slightly to be in line with our numerical results, we obtain that the onset of pebble accretion occurs at a planetesimal radius

$$
\begin{aligned}
R_{\mathrm{PA}} & \approx 0.67 v_{\mathrm{hw}} t_{\mathrm{X}}\left(\frac{t_{\mathrm{s}}}{t_{\mathrm{X}}}\right)^{0.28} \quad\left(t_{\mathrm{s}} \gg t_{\mathrm{X}}\right) \\
& \approx 520 \mathrm{~km}\left(\frac{v_{\mathrm{hw}}}{50 \mathrm{~m} \mathrm{~s}^{-1}}\right)\left(\frac{\rho_{\bullet}}{\mathrm{g} \mathrm{cm}^{-3}}\right)^{-0.36}\left(\frac{r}{\mathrm{AU}}\right)^{0.42} \tau_{\mathrm{s}}^{0.28}
\end{aligned}
$$

where the latter expression is valid for particles of $\tau_{\mathrm{s}}=t_{\mathrm{s}} \Omega<1$. Equation (24) agrees well with our numerical results.

\footnotetext{
7 Gravitational effects on the gas are of minor concern as long as the gas thermal motions are larger than the surface escape velocity.
} 


\section{Conclusions}

We identified the onset of pebble accretion by finding the collision factor for $1-10^{3} \mathrm{~km}$ planetesimals at 1,3 , and $10 \mathrm{AU}$ orbital distance for pebble sizes in the range of $0.01-30 \mathrm{~cm}$. To investigate the influence of the flow pattern in the vicinity of the planetesimal, we conducted numerical integrations for both potential and Stokes flow. From our numerical integrations and analytical fits we calculated a growth timescale on which the planetesimal mass $e$-folds by sweep-up of pebbles, investigating thereby the conditions under which small planetesimals can grow large enough for pebble accretion to become important. We conclude the following from our study:

1. Gravitational interactions between pebbles and planetesimals ensure that the collisional efficiency factors never become zero, even for the smallest particles.

2. Peak growth timescales for planetesimal sweep-up of pebble-sized particles occur at $\sim 100 \mathrm{~km}$, corresponding roughly to the point where the headwind velocity equals the surface escape velocity of the planetesimal. This transition size therefore decreases when the disk headwind is lower, for instance, for a colder disk. For very small particles (very small stopping times) peak growth timescales are longer because of aerodynamic deflection.

3. For Stokes flow, where the gas velocities vanish at the planetesimal surface, encounter times increase for smaller pebbles, increasing the likelihood to be captured by gravitational settling. In contrast, pebbles are centrifugally ejected in potential flow, strengthening the aerodynamic barrier.

4. A critical particle stopping time of $t_{\mathrm{X}} \approx 10^{3} \mathrm{~s}$ (see Eq. (22)) distinguishes between the slow pebble accretion regime, where particles suffer from aerodynamic deflection, and the fast pebble accretion regime, where growth proceeds by ballistic encounters (Safronov focusing), before transitioning to the settling regime (pebble accretion).

5. The onset of pebble accretion occurs when the planetesimal radius equals $R_{\mathrm{PA}}$ (Eq. (24)). This is followed by an abrupt increase in the collision rate. Pebble accretion starts at larger planetesimal sizes at increasing distance from the star.

6. At $\sim 1$ AU orbital distances, growth timescales are shorter than the disk lifetime of 10 Myr for any planetesimal size, provided pebble sizes are $>0.1 \mathrm{~cm}$. For small planetesimals (below $100 \mathrm{~km}$ ) growth primarily depends on the collisional outcome (sticking, bouncing, or fragmentation) of the pebble-planetesimal collision.

7. In the outer disk the maximum growth timescale exceed 10 Myr. Therefore, planetary seeds must have formed from planetesimals large enough to gravitationally attract pebblesized particles: $R \gg 100 \mathrm{~km}$ (Safronov focusing) or the even faster pebble accretion, whence $R>R_{\mathrm{PA}}$.

Acknowledgements. The authors would like to thank Sebastiaan Krijt, Carsten Dominik, Tristan Guillot, Ralph Wijers, Lucia Klarmann for useful discussions and the referee, Anders Johansen, for an insightful report. C.W.O. is supported by the Netherlands Organization for Scientific Research (NWO).

\section{References}

Andrews, S. M., Wilner, D. J., Hughes, A. M., Qi, C., \& Dullemond, C. P. 2009, ApJ, 700, 1502

Bagnold, R. A. 1936, Proc. R. Soc. Lond. A, 157, 594

Balbus, S. A., \& Hawley, J. F. 1991, ApJ, 376, 214

Batchelor, G. K. 1967, An introduction to fluid dynamics (Cambridge University Press)

Beard, K. V., \& Grover, S. N. 1974, J. Atmos. Sci., 31, 543

Birnstiel, T., Klahr, H., \& Ercolano, B. 2012, A\&A, 539, A148

Bitsch, B., Lambrechts, M., \& Johansen, A. 2015, A\&A, 582, A112

Blum, J., \& Wurm, G. 2008, ARA\&A, 46, 21

Chambers, J. E. 2014, Icarus, 233, 83

Clayton, D. D., \& Nittler, L. R. 2004, ARA\&A, 42, 39

Cuzzi, J. N. 2004, Icarus, 168, 484

Eshagh, M. 2005, J. Earth Space Phys., 31, 1

Fehlberg, E. 1969, Nasa Technical Report, NASA TR R-315

Guillot, T., Ida, S., \& Ormel, C. W. 2014, A\&A, 572, A72

Güttler, C., Blum, J., Zsom, A., Ormel, C. W., \& Dullemond, C. P. 2010, A\&A, 513, A56

Hayashi, C., Nakazawa, K., \& Nakagawa, Y. 1985, in Protostars and Planets II, eds. D. C. Black, \& M. S. Matthews (Tuscon: Univ. of Arizona Press), 1100

Homann, H., Guillot, T., Bec, J., et al. 2015, A\&A, submitted

Johansen, A., Oishi, J. S., Low, M., et al. 2007, Nature, 448, 1022

Johansen, A., Youdin, A., \& Mac Low, M. 2009, ApJ, 704, L75

Johansen, A., Youdin, A. N., \& Lithwick, Y. 2012, A\&A, 537, A125

Johansen, A., Jacquet, E., Cuzzi, J. N., Morbidelli, A., \& Gounelle, M. 2015a, ArXiv e-prints [arXiv: 1505.02941]

Johansen, A., Mac Low, M.-M., Lacerda, P., \& Bizzarro, M. 2015b, Sci. Adv., 1,15109

Kretke, K. A., \& Levison, H. F. 2014, AJ, 148, 109

Krijt, S., Ormel, C. W., Dominik, C., \& Tielens, A. G. G. M. 2015, A\&A, 574, A83

Krijt, S., Ormel, C. W., Dominik, C., \& Tielens, A. G. G. M. 2016, A\&A, 586, A20

Lambrechts, M., \& Johansen, A. 2012, A\&A, 544, A32

Lambrechts, M., \& Johansen, A. 2014, A\&A, 572, A107

Lambrechts, M., Johansen, A., \& Morbidelli, A. 2014, A\&A, 572, A35

Levison, H. F., Kretke, K. A., \& Duncan, M. J. 2015, Nature, 524, 322

Meisner, T., Wurm, G., Teiser, J., \& Schywek, M. 2013, A\&A, 559, A123

Michael, D. H., \& Norey, P. W. 1969, J. Fluid Mech., 37, 565

Morbidelli, A., Lambrechts, M., Jacobson, S., \& Bitsch, B. 2015, Icarus, 258, 418

Nakagawa, Y., Sekiya, M., \& Hayashi, C. 1986, Icarus, 67, 375

Ormel, C. W., \& Klahr, H. H. 2010, A\&A, 520, A43

Ormel, C. W., \& Kobayashi, H. 2012, ApJ, 747, 115

Ormel, C. W., Cuzzi, J. N., \& Tielens, A. G. G. M. 2008, ApJ, 679, 1588

Pfalzner, S., Steinhausen, M., \& Menten, K. 2014, ApJ, 793, L34

Ribas, Á., Bouy, H., \& Merín, B. 2015, A\&A, 576, A52

Ricci, L., Testi, L., Natta, A., \& Brooks, K. J. 2010, A\&A, 521, A66

Safronov, V. S. 1972, Evolution of the protoplanetary cloud and formation of the earth and planets (Keter Publishing House)

Sato, T., Okuzumi, S., \& Ida, S. 2015, A\&A, submitted [arXiv: 1512.02414]

Sekiya, M., \& Takeda, H. 2003, Earth, Planets, and Space, 55, 263

Sellentin, E., Ramsey, J. P., Windmark, F., \& Dullemond, C. P. 2013, A\&A, 560, A96

Shao, Y., \& Lu, H. 2000, J. Geophys. Res., 105, 22437

Slinn, W. G. N. 1976, Geophys. Res. Lett., 3, 21

Teiser, J., \& Wurm, G. 2009, MNRAS, 393, 1584

Testi, L., Birnstiel, T., Ricci, L., et al. 2014, Protostars and Planets VI, 339

Weidenschilling, S. J. 1977a, MNRAS, 180, 57

Weidenschilling, S. J. 1977b, Ap\&SS, 51, 153

Whipple, F. L. 1972, in Proc. Symp., From Plasma to Planet, ed. A. Elvius, 211

Windmark, F., Birnstiel, T., Güttler, C., et al. 2012, A\&A, 540, A73

Yasui, C., Kobayashi, N., Tokunaga, A. T., \& Saito, M. 2014, MNRAS, 442, 2543

Youdin, A. N., \& Goodman, J. 2005, ApJ, 620, 459

Youdin, A. N., \& Lithwick, Y. 2007, Icarus, 192, 588 